\title{
Estratégias para o condicionamento comportamental em pacientes com transtorno do espectro autista durante o atendimento odontológico
}

\author{
Strategies for behavioral conditioning in patients with autistic spectrum disorder during dental \\ care \\ Estrategias de condicionamiento conductual en pacientes con trastorno del espectro autista durante \\ la atención odontológica
}

Recebido: 09/11/2021 | Revisado: 18/11/2021 | Aceito: 23/11/2021 | Publicado: 04/12/2021

\author{
Amanda Cristina Silva \\ ORCID: https://orcid.org/0000-0002-3056-032X \\ Centro Universitário de Patos de Minas, Brasil \\ E-mail:amandacristinas@unipam.edu.br \\ Carolina Silva Pereira \\ ORCID: https://orcid.org/0000-0003-2020-3932 \\ Centro Universitário de Patos de Minas, Brasil \\ E-mail:carolinasp@unipam.edu.br \\ Gabriella Moreira dos Anjos \\ ORCID: https://orcid.org/0000-0002-5351-6540 \\ Centro Universitário de Patos de Minas, Brasil \\ E-mail:gabriellamoreira@unipam.edu.br \\ Daniella Cristina Borges \\ ORCID: https://orcid.org/0000-0002-5424-5367 \\ Centro Universitário de Patos de Minas, Brasil \\ E-mail:daniellacborges@unipam.edu.br \\ Helvécio Maragon Júnior \\ ORCID: https://orcid.org/0000-0002-9709-6795 \\ Centro Universitário de Patos de Minas, Brasil \\ E-mail:helveciomjr@unipam.edu.br \\ Leonardo Bíscaro Pereira \\ ORCID: https://orcid.org/ 0000-0001-9790-9082 \\ Centro Universitário de Patos de Minas, Brasil \\ E-mail: leonardobiscaro@unipam.edu.br
}

\begin{abstract}
Resumo
Transtorno do Espectro Autista (TEA) é um distúrbio do neurodesenvolvimento com etiologia indefinida e graus variados de severidade. Caracterizado por déficits na comunicação e na interação social, além de padrões restritivos de comportamento. $\mathrm{O}$ atendimento odontológico desses pacientes é desafiador, pois suas manifestações clínicas são complexas e variadas. Isso ocorre porque o ambiente odontológico e, o próprio atendimento, apresentam potenciais gatilhos para alterações comportamentais nos portadores de TEA, visto que eles possuem uma maior sensibilidade aos estímulos presentes no consultório. Este estudo trata-se de uma revisão de literatura cujo objetivo é identificar as estratégias de manejo comportamental empregadas pelo dentista frente aos desafios encontrados no paciente autista. Foi realizado um levantamento bibliográfico de artigos indexados nas bases eletrônicas PUBMED, SCIELO e BVS, utilizando os descritores "autism spectrum disorder", “autism”, "oral health", "behavior” e "dental care”. Foram selecionados artigos encontrados na íntegra considerando os últimos 10 anos, excluindo aqueles que não traziam um bom embasamento sobre o tema. O manejo odontológico do paciente com TEA requer individualização e a compreensão do seu comportamento, englobando diferentes técnicas como: dizer-mostrar-fazer, sessões curtas, eliminação de estímulos sensoriais estressantes, controle de voz, modelação, além das abordagens educacionais PECS, ABA e TEACCH. Conclui-se que, apesar dos desafios, um tratamento odontológico de qualidade pode ser realizado com o uso de abordagens personalizadas, para isso, é necessário que o Cirurgião-Dentista conheça esses recursos e domine a técnica.
\end{abstract}

Palavras-chave: Transtorno do espectro autista; Autismo; Comportamento; Tratamento odontológico.

\section{Abstract}

Autism Spectrum Disorder (ASD) is a neurodevelopmental disorder with undefined etiology and varying degrees of severity. It is characterized by deficits in communication and social interaction, and restrictive behavior patterns. The dental care of these patients is challenging, because their clinical manifestations are complex and varied. This occurs 
because the dental environment, and the service itself, have potential triggers for behavioral changes in people with ASD, since they have a greater sensitivity to the stimuli present in the dental office. This study is a literature review that aims to identify the behavioral management strategies employed by the dentist when facing the challenges faced by the autistic patient. A bibliographic survey of articles indexed in the electronic databases PUBMED, SCIELO, and BVS was carried out using the descriptors "autism spectrum disorder", "autism", "oral health", "behavior", and "dental care". Articles from the last 10 years were selected in their entirety, excluding those that were not well grounded on the theme. The dental management of the patient with ASD requires individualization and understanding of his behavior, encompassing different techniques such as: tell- show-do, short sessions, elimination of stressful sensory stimuli, voice control, modeling, in addition to the educational approaches PECS, ABA and TEACCH. It is concluded that, despite the challenges, quality dental treatment can be accomplished with the use of personalized approaches, for this, it is necessary that the dental surgeon knows these resources and master the technique.

Keywords: Autistic spectrum disorder; Autism; Behavior; Dental treatment.

\section{Resumen}

El Trastorno del Espectro Autista (TEA) es un trastorno del neurodesarrollo de etiología indefinida y con distintos grados de gravedad. Se caracteriza por déficits en la comunicación y la interacción social, además de patrones de comportamiento restrictivos. El cuidado dental de estos pacientes es un reto porque sus manifestaciones clínicas son complejas y variadas. Esto ocurre porque el entorno odontológico y el propio servicio tienen potenciales desencadenantes de cambios conductuales en las personas con TEA, ya que son más sensibles a los estímulos en la consulta. Este estudio es una revisión de la literatura que tiene como objetivo identificar las estrategias de gestión del comportamiento empleadas por los dentistas para hacer frente a los desafíos que enfrentan los pacientes autistas. Se realizó un estudio bibliográfico de los artículos indexados en las bases de datos electrónicas PUBMED, SCIELO y BVS utilizando los descriptores "autism spectrum disorder", "autism", "oral health", "behavior" y "dental care". Los artículos se seleccionaron en su totalidad teniendo en cuenta los últimos 10 años, excluyendo aquellos que no aportaban una buena base sobre el tema. El manejo odontológico del paciente con TEA requiere la individualización y comprensión de su comportamiento, abarcando diferentes técnicas como: decir-mostrar-hacer, sesiones cortas, eliminación de estímulos sensoriales estresantes, control de la voz, modelado, además de los enfoques educativos PECS, ABA y TEACCH. Se concluye que, a pesar de los retos, se puede realizar un tratamiento dental de calidad con el uso de enfoques personalizados, para ello, es necesario que el cirujano dental conozca estos recursos y domine la técnica.

Palabras clave: Trastorno del espectro autista; El autismo; Comportamiento; Tratamiento dental.

\section{Introdução}

O conceito de paciente com necessidade especial (PNE) é definido como todo indivíduo, adulto ou criança, que apresenta algum desvio de normalidade, permanente ou temporário, podendo ser de ordem física, mental, social ou emocional, requerendo atenção e abordagens especiais que atendam a suas demandas de acordo com suas necessidades específicas (Lei ${ }^{\circ}$ 13.146, de 6 de julho de 2015).

Dentre as alterações definidas como necessidades especiais está o Transtorno do Espectro Autista (TEA), um distúrbio do neurodesenvolvimento caracterizado por um grupo principal de fatores definidores que compreendem déficits na comunicação verbal e não verbal, convívio social afetado, além de padrões restritos e repetitivos de comportamento e reações imprevisíveis a estímulos ambientais (American Psychiatric Association, 2014).

Essa condição recebe o nome de espectro, pois pode apresentar diferentes níveis de comprometimento classificados em graus leve, moderado ou severo, sendo sua incidência maior no gênero masculino. Sua etiologia ainda não é definida, contudo acredita-se que se dê através de bases multifatoriais (American Psychiatric Association, 2014).

O autismo geralmente tem o seu diagnóstico na infância, podendo apresentar sinais patognomônicos antes mesmo dos três anos de idade e persistindo até a idade adulta. Esse diagnóstico é basicamente clínico, baseado na avaliação qualitativa de padrões comportamentais (Gomes et al., 2015). Os critérios empregados para diagnosticar o transtorno são descritos no Manual Estatístico e Diagnóstico da Associação Americana de Psiquiatria, o DSM-5 (American Psychiatric Association, 2014).

No entanto, a ampla variedade de expressões comportamentais, somada à inexistência de uma etiologia estabelecida e a falta de serviços especializados nessa patologia, torna esse diagnóstico um desafio. É de grande importância a detecção 
precoce e o início de intervenções terapêuticas o mais cedo possível. O atraso do diagnóstico e do tratamento pode gerar prejuízos no desenvolvimento da criança (Jendreieck, 2014, Bosa, Zanon \& Backes, 2017).

Estudos epidemiológicos realizados na última década mostraram um aumento significativo na prevalência do TEA, que alcançou nos últimos anos o índice de 2\% da população (Hill, Zuckerman \& Fombonne, 2015; Maenner et al., 2020). Isso sugere que a probabilidade de Cirurgiões-Dentistas encontrarem pacientes autistas em seu consultório odontológico é alta (Zink et al., 2016). Com essa demanda, faz-se necessário uma maior conscientização dos odontólogos quanto ao desenvolvimento de abordagens odontológicas adequadas para esse público (Fonseca et al., 2010).

Características clínicas dos pacientes autistas, como percepção sensório-motora exacerbada, problemas de atenção, ansiedade, controle emocional, dificuldade de compreensão e hipossensibilidade ou indiferença à dor, representam desafios frente ao atendimento odontológico (Delli et al., 2013). Com base nisso, para que seja possível realizar os procedimentos adequados direcionados às especificidades desses pacientes, é necessário aplicar manobras e técnicas individualizadas que garantam um contato menos traumático e atendam todas as demandas odontológicas de maneira eficiente (Cagetti et al., 2015).

O presente estudo propõe, através de uma revisão da literatura existente, descrever as abordagens clínicas de manobras e técnicas básicas de condicionamento empregadas pelo Cirurgião-Dentista para o gerenciamento comportamental do paciente portador do TEA. Fornecendo aos graduandos em odontologia e Cirurgiões-Dentistas subsídios para o atendimento mais humanizado, ético, com condutas individualizadas e adaptação profissional aos pacientes com TEA.

\section{Metodologia}

Para elaboração desse estudo foi realizada uma revisão narrativa da literatura a respeito do manejo comportamental dos pacientes com transtorno do espectro autista (TEA). Para o seu desenvolvimento, foi feito um levantamento bibliográfico utilizados livros, artigos científicos e material eletrônico das bases de dados Biblioteca Virtual de Saúde (BVS), PUBMED e SCIELO. Para otimizar e aprimorar as buscas foram utilizados os Descritores em Ciência da Saúde (DECS): "transtorno do espectro autista", "autismo", "saúde bucal", "comportamento", "tratamento odontológico" e no inglês "autism spectrum disorder", "autism", "oral health", "behavior" e "dental care", que foram associados por meio dos operadores booleanos "e" e "ou" e seus correspondentes em inglês.

Foram incluídos no estudo artigos publicados no período entre junho 2011 e junho 2021, que apresentaram discussões a respeito das técnicas de manejo comportamental e dificuldades nos atendimentos odontológicos de pacientes autistas. Excluiu-se do estudo, artigos que não estavam disponíveis na íntegra, opiniões de especialistas, dissertações e resumos ainda que publicados em revista indexada.

\section{Resultados}

\subsection{Autismo}

O termo autismo tem sua origem do grego "autós", que se refere a si mesmo, mais o sufixo "ismos", indicando ação ou estado; assim, se caracteriza pelo ato do indivíduo de introverter-se para seu próprio interior. Foi empregado pela primeira vez pelo psiquiatra suíço Plouller, em 1906, mas foi difundido somente em 1911, por Eugene Bleuder, que buscava descrever a fuga da realidade e o retraimento interior dos pacientes acometidos pela esquizofrenia (Volkmar \& Wiesner, 2019).

O autismo foi citado novamente em 1943, quando o médico austríaco Leo Kanner sistematizou a cuidadosa observação de um grupo de onze crianças que apresentavam dificuldades de comunicação e relacionamento, na qual denominou de Distúrbio Autístico do Contato Afetivo (Zink et al., 2016). Outros autores desenvolveram estudos sobre o assunto e suas descobertas vêm contribuindo, até os dias atuais, para uma melhor compreensão desse transtorno. 
De acordo com o Manual Diagnóstico e Estatístico de Transtornos Mentais (DSM-5), o TEA é compreendido como um transtorno do neurodesenvolvimento e pode ser definido da seguinte forma:

O transtorno do espectro autista caracteriza-se por déficits persistentes na comunicação social e na interação social em múltiplos contextos, incluindo déficits na reciprocidade social, em comportamentos não verbais de comunicação usados para interação social e em habilidades para desenvolver, manter e compreender relacionamentos. Além dos déficits na comunicação social, o diagnóstico do transtorno do espectro autista requer a presença de padrões restritos e repetitivos de comportamento, interesses ou atividades (American Psychiatric Association, 2014, p. 31).

O TEA é uma desordem que ocorre durante o desenvolvimento embrionário, não sendo possível diagnosticá-lo no período pré-natal. Sua sintomatologia não surge de modo repentino, é um distúrbio que se desenvolve ao longo da vida. Normalmente, antes dos 30 meses de idade, o autista inicia a manifestação de comportamentos distintos, tais como obsessão por objetos e comportamentos repetitivos, falta de interesse pelo contato dos pais, dificuldade ao comunicar-se com outras pessoas (Roja, Rivera \& Nilo, 2019).

Não existe uma causa determinada para o desenvolvimento do autismo. As evidências científicas disponíveis indicam a existência de múltiplas causas, incluindo fatores genéticos, imunológicos, alterações neuropsicológicas, complicações neonatais ou perinatais, desequilíbrios metabólicos e aspectos ambientais (Mangione et al., 2020).

O espectro autista é observado em todas as etnias e classes socioeconômicas, sendo considerado atualmente um dos transtornos do neurodesenvolvimento mais comuns. Há relatos de que uma em cada 54 crianças dos EUA apresentam o transtorno, sendo que a prevalência é quatro vezes maior em meninos do que em meninas na faixa etária dos 8 anos de idade. Apesar de a condição ser mais frequente no sexo masculino, a deficiência intelectual é mais acentuada nas meninas (Centers for disease control and prevention, 2020).

Não existe, atualmente, um marcador biológico para a identificação do TEA em exames laboratoriais. No entanto, o diagnóstico pode ser feito por meio da interação de informações vindas de uma minuciosa entrevista clínica, entrevistas com os responsáveis, observação do paciente e um exame médico e neurológico detalhado para excluir comorbidades médicas e psiquiátricas associadas. Os critérios utilizados para diagnosticar o autismo estão descritos no Manual Diagnóstico e Estatístico de Transtornos Mentais (DSM-V), e baseiam-se em evidências do comprometimento da interação e comunicação sociais e na presença de um repertório restrito de comportamentos, atividades e interesses (American Psychiatric Association, 2014).

Nas diferentes expressões do quadro clínico, diversos sinais e sintomas podem estar ou não presentes. Todavia, a última versão do DSM (DSM-V) propôs uma nova classificação, que desconsidera as categorias Autismo, Síndrome de Asperger, Transtorno Desintegrativo e Transtorno Global do Desenvolvimento Sem Outra Especificação. Os subtipos comportamentais citados foram reunidos numa única denominação e passam a receber o diagnostico único de TEA. As avaliações priorizam a intensidade dos sintomas, que podem ser leves, moderados ou severos (American Psychiatric Association, 2014).

O TEA apresenta características semelhantes a algumas condições, como atraso mental, transtornos de ansiedade e transtorno do apego reativo (TAR), distúrbio do desenvolvimento da linguagem do tipo repetitivo, síndrome de Rett, transtorno obsessivo-compulsivo (TOC) e a esquizofrenia, tornando-se imprescindível um diagnóstico diferencial (Araújo et al., 2019).

Dados mais recentes indicam que as estatisticas de prevalência do transtorno estão aumentando, mas se discute em que medida isso advém da mudança dos critérios diagnósticos, de diferenças metodológicas nos estudos científicos, de um melhor reconhecimento do transtorno por profissionais de saúde ou de aumento real na sua fequência (American Psychiatric Association, 2014). 


\subsection{Tratamento odontológico no paciente autista}

Os portadores de TEA apresentam necessidades de saúde complexas e carecem de um tratamento multidisciplinar, para que haja uma abordagem humanizada e capacitada, que incluem serviços de promoção, cuidados e reabilitação da saúde. As intervenções direcionadas aos portadores do espectro autista devem seguir medidas amplas que tornem seus ambientes físicos e sociais mais acessíveis, inclusivos e acolhedores (Brasil, 2017).

O comportamento autístico representa um grande desafio para os profissionais frente ao tratamento odontológico. São pacientes que apresentam déficit na comunicação, incapacidade de controlar emoções, movimentos repetitivos, hiperatividade e déficit de atenção (Delli et al., 2013). Além disso, os autistas também apresentam a percepção sensorial alterada, o que pode estimular alterações comportamentais, através das luzes fluorescentes, equipamentos que geram ruídos, materiais de texturas e aromas desconhecidos, dentre outros estímulos que são facilmente encontrados no consultório odontológico. Portanto, é de suma importância que o Cirurgião-Dentista identifique e minimize estes fatores que geram um comportamento negativo (Stein et al., 2011).

Encontrar novas alternativas de intervenção e acolhimento destes indivíduos deve ser uma busca constante de todos que trabalham com o paciente portador do espectro autista. Diante disso, podem ser empregadas estratégias para melhorar a comunicação com o paciente, levando em conta recursos verbais, não verbais, sensoriais, entre outros (Ganz et al., 2012).

O manejo comportamental é tanto uma arte quanto uma ciência, não é uma aplicação de técnicas, mas um método contínuo que implica em desenvolver o relacionamento entre o paciente e o profissional, visando à construção de um vínculo que aliviará o medo e a ansiedade do paciente (Massara \& Rédua,2013). Desta forma, faz-se necessário a orientação dos profissionais odontólogos para proporcionarem cuidados de saúde bucal a esses indivíduos, utilizando de técnicas não farmacológicas.

A abordagem comportamental dos portadores do TEA pode ser realizada através de diversos métodos e técnicas, dependendo das características individuais de cada paciente. As principais técnicas básicas utilizadas são: dizer-mostrar-fazer, distração, dessensibilização, controle de voz, reforço positivo, eliminação de estímulos sensoriais estressantes e modelação. Além disso, diversas abordagens educacionais têm sido desenvolvidas com o objetivo de promover o desenvolvimento social das pessoas com TEA, sendo assim, são capazes de auxiliar o Cirurgião-Dentista durante o tratamento do paciente autista. Entre elas estão: Picture Exchange Communication System (PECS); Applied Behavior Analysis (ABA), o Treatment and Education of Autistic and Related Communication Handicapped Children (TEACCH), entre outros (Amaral et al., 2012).

A técnica dizer-mostrar-fazer consiste em apresentar ao paciente elementos que serão utilizados durante seu atendimento odontológico, através de uma explicação verbal com a linguagem apropriada ao desenvolvimento psicológico do autista. Isso ajudará a reduzir o medo e a ansiedade e a familiarizar a criança com a equipe e o ambiente da consulta (Delli et al., 2013). Na técnica da distração o principal objetivo é desviar a atenção do paciente para evitar o foco no procedimento odontológico. Para isso, podem ser adotadas estratégias de manejo utilizando músicas, vídeos e histórias (Nelson et al., 2014).

A dessensibilização é uma técnica de abordagem gradual, onde o portador do espectro autista é exposto repetidas vezes ao ambiente odontológico, visando sua adaptação e cooperação durante as visitas ao dentista. Ao realizar o controle de voz o dentista deverá alterar o volume, o tom e o ritmo da voz de forma controlada buscando recuperar a atenção do paciente. Tendo em conta que indivíduos com TEA apresentam hipersensibilidade sensorial, deve-se optar por usar uma voz calma, não sendo aconselhável, o aumento do volume de voz, pois poderá ter o efeito oposto, agravando o comportamento não colaborativo. O reforço positivo consiste em recompensar o paciente por apresentar bons comportamentos através de elogios, sorrisos, demonstrações de afeto e/ou prêmios. O objetivo é incentivar comportamentos positivos, fazendo com que se repitam nas próximas consultas (Cameron \& Widmer, 2012, Delli et al., 2013, Nelson et al., 2014). 
Eliminação de estímulos sensoriais estressantes: é importante que a equipe odontológica fique atenta aos estímulos que despertem comportamentos negativos no paciente autista, desse modo deve ser realizada uma adequação sensorial do ambiente clínico buscando minimizar esse incomodo emocional (Delli et al., 2013, Nelson et al., 2014).

$\mathrm{Na}$ técnica da modelação o paciente com medo e/ou ansioso observa o tratamento de um indivíduo colaborador e, assim, os comportamentos favoráveis servem como exemplo para o portador do espectro autista (Nelson et al., 2014).

Nos últimos anos houve um avanço na abordagem de pacientes com necessidades especiais frente ao atendimento odontológico, incluindo os portadores do espectro autista. Essa evolução está relacionada à aplicação de técnicas psicopedagógicas como PECS, ABA e TEACCH, que empregam recursos visuais, corporais e até mesmo a análise de comportamento, buscando uma melhor interação e comunicação com o paciente (Cagetti et al., 2015).

As técnicas terapêuticas propostas para os indivíduos com TEA são focadas em um atendimento mais humanizado e diferenciado, apresentando resultados positivos para os pacientes, familiares, cuidadores e para os Cirurgiões-Dentistas. Porém, os estudos sobre o atendimento odontológico para tais pacientes são reduzidos, diante disso, é necessária a realização de mais pesquisas cientificas para mobilizarem-se e estarem preparados para a inserção destas novas metodologias em suas práticas (Stein Duker et al., 2019).

\subsection{Sedação consciente}

As técnicas de abordagens comportamentais básicas são a primeira opção para o controle da ansiedade antes de iniciar um tratamento odontológico em pacientes com TEA porém, em algumas situações, elas necessitam estar associadas a outras estratégias. Em pacientes autistas não colaborativos, ou com índice de comprometimento severo, a sedação consciente torna-se uma opção. Este método reduz a ansiedade e o medo, aumenta o limiar de dor e consequentemente facilita o controle comportamental do paciente. A terapêutica da sedação consiste em uma leve depressão do nível de consciência por meio do uso de fármacos como óxido nitroso, benzodiazepínicos e anti-histamínicos. As drogas sedativas mais comumente utilizadas são: óxido nitroso, midazolam, prometazina, hidroxina, hidrato de cloral e diazepam. Entretanto, apenas Cirurgiões-Dentistas habilitados podem realizar a técnica da sedação consciente com óxido nitroso inalatório, realizando o devido monitoramento do paciente (Fiorillo, 2019, Picciani et al., 2019).

Para correta execução da técnica de sedação consciente é necessário se atentar às condições sistêmicas do paciente e ter conhecimento se o mesmo faz uso de alguma medicação, para evitar o risco de complicações devido às interações medicamentosas. Além disso, o método requer uma explicação detalhada e o consentimento dos pais ou responsáveis para sua realização, gerando assim uma confiança no trabalho a ser desenvolvido pelos profissionais (Fiorillo, 2019).

\section{Discussão}

A partir da revisão de literatura acerca da abordagem odontológica a pacientes com TEA, foi possível identificar que há poucas publicações que abordam essa temática, o que dificulta o plano de tratamento dos Cirurgiões-Dentistas (Nelson et al., 2014). Mesmo o profissional ciente de que o paciente necessite de abordagem individualizada, faz-se necessário informações adicionais e científicas em maior número e acesso (Picciani et al., 2019). Diante disso, é de suma importância conhecer as dificuldades enfrentadas pelos pacientes diagnosticados com TEA para o acesso ao atendimento odontológico.

Dentre as limitações encontradas, as mais relatadas na literatura são: alterações do comportamento relacionadas à interação social, dificuldade em estabelecer contato visual, alterações na percepção sensorial, déficit na comunicação e limitações motoras (Amaral et al., 2012, Araújo et al., 2019, Delli et al., 2013). Diante da prevalência das alterações comportamentais supracitadas, técnicas avançadas como estabilização protetora, sedação e anestesia geral são muito utilizadas 
nestes pacientes. Todavia, apesar dessas técnicas atenderem suas necessidades odontológicas imediatas, elas podem predispor o paciente a um risco físico e psicológico, além de não estimularem o paciente quanto aos cuidados bucais a longo prazo (Nelson, 2014). Portanto, só devem ser utilizadas em último caso, quando não for possível realizar o atendimento utilizando de abordagens comportamentais básicas (Amaral et al., 2012, Delli et al., 2013, Nelson, 2014).

As estratégias de orientação comportamental básicas têm como objetivo desenvolver a harmonia, diminuir a ansiedade e fornecer um atendimento odontológico de qualidade, ao mesmo tempo em que se constroem uma relação de vínculo entre os profissionais envolvidos e o paciente (Delli et al., 2013).

Não existe um protocolo de manejo de comportamento aplicável a todos os autistas. Informações como o grau do transtorno, seus hábitos de higiene oral, estilo de comunicação, limitações, preferências, sensibilidades sensoriais e a presença de determinadas condições associadas podem orientar o Cirurgião-Dentista na escolha das melhores técnicas a serem utilizadas. Portanto, é recomendável que esses dados sejam coletados antes das intervenções odontológicas, através de uma anamnese criteriosa com os pais ou responsáveis pelo paciente (Amaral et al., 2012, Delli et al., 2013, Nelson et al., 2014).

É consenso na literatura que a familiarização do autista com o ambiente odontológico é primordial. Sendo assim, as primeiras visitas ao dentista podem ser simplesmente para explorar o consultório, a fim de realizar a dessensibilização do paciente quanto ao ambiente. Esta etapa envolve uma intervenção gradual, que leva tempo e para a qual não é possível prever quais pacientes obterão sucesso ou quantas consultas serão necessárias nessa fase. Sessões curtas e bem organizadas devem ser planejadas e o tempo de espera na recepção deve ser minimizado para evitar transtornos, pois esses pacientes possuem tempo de atenção limitado. Além disso, indivíduos com TEA apresentam aversão a mudanças, sendo necessário estabelecer rotinas, agendando as consultas no mesmo dia da semana, mantendo o horário e a mesma equipe odontológica (Amaral et al. 2012, Delli et al., 2013, Gandhi \& Klein, 2014; Nelson et al., 2014).

Outra estratégia adotada é o controle de voz, no entanto, a dificuldade de compreensão da linguagem pode ser um obstáculo. Para-se obter sucesso nessa técnica as ordens devem ser claras e objetivas, empregando frases de conhecimento do paciente, evitando o uso de figuras de linguagem. Se o paciente for capaz de entender a comunicação não verbal, o uso de expressões faciais pode ser utilizado (Amaral et al. 2012; Delli et al. 2013; Nelson et al., 2014).

Sabendo que os pacientes com TEA têm hipersensibilidade sensorial, é essencial diminuir a exposição a esses estímulos irritantes. O objetivo é reduzir o desconforto e assim evitar comportamentos negativos que possam surgir em consequência dessa percepção. Para isso, podem ser adotadas medidas experimentais utilizando luzes relaxantes, uso de óculos escuros, músicas, videoclipes e objetos da preferência do paciente, buscando sua distração e consequente comportamento positivo. A técnica da modelagem mostra mais eficiência quando a demonstração do atendimento é feita em pessoas próximas ao autista. O objetivo é que o paciente repita as boas condutas da pessoa de sua confiança (Amaral et al., 2012, Delli et al., 2013, Nelson et al., 2014).

Outra estratégia muito utilizada é a recompensa ao comportamento positivo, que pode ser associada a todas as técnicas já mencionadas. Visa à utilização de elogios, expressões positivas e até mesmo prêmios. É de grande valia conhecer as preferências do paciente, identificando se ele possui algum tipo de objeto de estima, ao qual se dirige em situações de estresse ou ansiedade, que possa ser utilizado como recompensa (Delli et al., 2013; Gandhi \& Klein, 2014; Nelson et al., 2014).

$\mathrm{O}$ método TEACCH ou Tratamento e educação para crianças autistas e com distúrbios correlacionados à comunicação é uma das abordagens mais utilizadas e eficazes, que emprega o uso de imagens, fotografias, histórias sociais e vídeos adaptados para demonstrar ao autista o que deve fazer, quando e como (Cagetti et al., 2015). Na odontologia, o TEACCH é aplicado visando à compreensão e a formação de hábitos, ajudando na cooperação do paciente nas consultas clínicas e até mesmo nos cuidados orais em sua rotina (Amaral et al., 2012). 
Outro sistema de instrução com base visual é o método PECS, baseado na troca de imagens fotos e vídeos, que facilitam a comunicação com pacientes não verbais e/ou não fluentes. É abordado na odontologia para realizar demonstrações de etapas clínicas e orientações de higiene bucal (Amaral et al., 2012, Gandhi \& Klein, 2014.).

A análise aplicada do comportamento (ABA), também é muito utilizada para o planejamento de intervenções de tratamento para pessoas com TEA, priorizando o desenvolvimento de habilidades sociais, motoras e na área da comunicação (Cagetti et al., 2015). No atendimento odontológico, o uso dessa abordagem auxilia nas práticas convencionais de manejo comportamental. É realizada em diferentes etapas, e o paciente é incentivado a adotar o comportamento por vontade própria (Hernandez \& Ikkanda, 2011, Cagetti et al., 2015).

A escolha por uma estratégia terapêutica deve ser baseada em informações claras a respeito de suas particularidades, grau do transtorno, a técnica a ser realizada e expectativas de resultados. Além disso, é necessário orientar e informar à família quanto às alternativas disponíveis, suas vantagens e limitações (Fernandes \& Amato, 2013).

\section{Conclusão}

O Transtorno do espectro autista apresenta grande importância para a sociedade atual, tendo em vista o aumento crescente de pacientes diagnosticados com autismo. Há a necessidade de se conhecer mais sobre o TEA e, para isso, é preciso pesquisar diferentes abordagens para melhor atendê-los. A melhor compreensão desse transtorno ajuda a evitar situações que poderiam causar danos físicos e psicológicos para os pacientes e familiares, durante o tratamento odontológico. Conclui-se que, apesar dos desafios, um tratamento odontológico de qualidade pode ser realizado com o uso de abordagens personalizadas e, para isso, é necessário que o Cirurgião-Dentista conheça esses recursos e domine as técnicas.

\section{Referências}

Amaral, C. O. F., Malacrida, V. H., Videira, F. C. H., Parizi, A. G. S., Oliveira, A. \& Straioto, F. G. (2012). Paciente autista: métodos e estratégias de condicionamento e adaptação para o atendimento odontológico. Arch Oral Res., 8(2), 51-143. https://doi.org/10.7213/aor.v8i2.23056

American Psychiatric Association (APA). (2014). DSM-5: Manual diagnóstico e estatístico de transtornos mentais. Artmed Editora. https://books.google.com.br/books?id=QL4rDAAAQBAJ\&printsec=frontcover\&hl=pt-BR\&source=gbs_ge_summary_r\&cad=0\#v=onepage\&q\&f=false

Araújo, L. A. Chaves, L. F. S., Loureiro, A. A., Alves, A. M. G., Lopes, A. M. C. S., Barros, J. C. R., Halpern, R., Cardoso, A. A., Veloso, C. F., CardosoMartins, C., Fernandes, F. D. M., Magalhães, M. L. \& Nogueira, M. F. (2019). Transtorno do Espectro do Autismo. Departamento científico de pediatria do desenvolvimento e comportamento. Sociedade brasileira de pediatria, 5, 1-24. https://www.sbp.com.br/fileadmin/user_upload/21775cMOTranstorno_do_Espectro_do_Autismo.pdf

Bosa, C. A., Zanon, R. B. \& Backes, B. (2017). Autismo: construção de um protocolo de avaliação do comportamento da criança -Protea-R. Revista Psicológica: Teoria e Prática, São Paulo, 18(1), 194-205. http://dx.doi.org/10.15348/1980-6906/psicologia.v18n1p194-205

Brasil. (2017). Organização Pan-Americana da Saúde. Folha informativa - Transtornos do espectro autista. https://www.paho.org/pt/search/r?keys=folh\%20a+informativa+transtornos+do+espectro+autista+Brasil

Cagetti, M. G., Mastroberardino, S., Campus, S., Olivari, B., Faggioli, R., Lenti, C., \& Strohmenger, L. (2015). Dental care protocol based on visual supports for children with autism spectrum disorders. Medicina oral, patologia oral y cirugia bucal, 20(5). https://doi.org/10.4317/medoral.20424

Cameron, A. C. \& Widmer, R. P. (2012). Manual de Odontopediatria (3a ed) Elsevier. https://br1lib.org/book/17416087/d85b3d?id= $17416087 \&$ secret $=\mathrm{d} 85 \mathrm{~b} 3 \mathrm{~d}$

Centers for disease control and prevention (CDC). $\quad$ (2017). Autism Spectrum $\quad$ Disorder https://www.cdc.gov/mmwr/volumes/69/ss/ss6904a1.htm?s_cid=ss6904a1_w

Delli, K., Reichart, P. A., Bornstein, M. M., \& Livas, C. (2013). Management of children with autism spectrum disorder in the dental setting: concerns, behavioural approaches and recommendations. Medicina oral, patologia oral y cirugia bucal, 18(6). https://doi.org/10.4317/medoral.19084

Fernandes, F. D. M. \& Amato, C. A. H. (2013). Análise de Comportamento Aplicada e Distúrbios do Espectro do Autismo: revisão de literatura. CoDAS, 25(3), 289-296. https://www.scielo.br/j/codas/a/vgGhzWvhgWXJXp5PrvBK9Nr/abstract/?lang=pt

Fiorillo L. (2019). Conscious Sedation in Dentistry. Medicina (Kaunas, Lithuania), 55(12), 778. https://doi.org/10.3390/medicina55120778 
Fonseca, A. L. A., Azzalis, L. A., Fonseca, F. L. A., \& Botazzo, C. (2010). Análise qualitativa das percepções de cirurgiões-dentistas envolvidos nos atendimentos de pacientes com necessidades especiais de serviços públicos municipais. Journal of Human Growth and Development, 20(2), 208-216. http://pepsic.bvsalud.org/scielo.php?script=sci_arttext\&pid=S0104-12822010000200004\&lng=pt\&tlng=pt.

Gandhi, R. P., \& Klein, U. (2014). Autism spectrum disorders: an update on oral health management. The journal of evidence-based dental practice, 14 Suppl, 115-126. https://doi.org/10.1016/j.jebdp.2014.03.002

Ganz, J. B., Davis, J. L., Lund, E. M., Goodwyn, F. D., \& Simpson, R. L. (2012). Meta-analysis of PECS with individuals with ASD: Investigation of targeted versus non-targeted outcomes, participant characteristics, and implementation phase. Research in Developmental Disorders, 33, 406-418. https://doi.org/10.1007/s10803-011-1212-2

Gomes, P. T. M., Lima, L. H. L., Bueno, M. K. G., Araújo, L. A. \& Souza, N. M. (2015). Autism in Brazil: a systematic review of family challenges and coping strategies. J Pediatr (RJ), 91 (2). https://doi.org/10.1016/j.jped.2014.08.009

Hernandez, P., \& Ikkanda, Z. (2011). Applied behavior analysis: behavior management of children with autism spectrum disorders in dental environments. Journal of the American Dental Association, 142(3), 281-287. https://doi.org/10.14219/jada.archive.2011.0167

Hill, A. P., Zuckerman, K. E., \& Fombonne, E. (2014). Epidemiology of autism spectrum disorders. Handbook of Autism and Pervasive Developmental Disorders, 4. https://doi.org/10.1002/9781118911389.hautc03

Jendreieck, C. D. O. (2014). Dificuldades encontradas pelos profissionais da saúde ao realizar diagnóstico precoce de autismo. Psicologia Argumento, Curitiba, 32(77), 153-158. https://doi.org/10.7213/psicol.argum.32.077.AO09

Lei $n^{o}$ 13.146, de 6 de julho de 2015. (2015). Institui a Lei Brasileira de Inclusão da Pessoa com Deficiência (Estatuto da Pessoa com Deficiência). Brasília, DF.

Maenner, M. J., Shaw, K. A., Baio, J., Washington, A., Patrick, M., DiRienzo, M. ... Dietz, P. M. (2020). Prevalence of Autism Spectrum Disorder Among Children Aged 8 Years - Autism and Developmental Disabilities Monitoring Network, 11 Sites, United States, 2016. (2020). MMWR Surveill Summ. 27;69(4):1-12. http://dx.doi.org/10.15585/mmwr.ss6904a1external icon

Mangione, F., Bdeoui, F., Monnier-Da Costa, A., \& Dursun, E. (2020). Autistic patients: a retrospective study on their dental needs and the behavioural approach. Clinical oral investigations, 24(5), 1677-1685. https://doi.org/10.1007/s00784-019-03023-7

Massara, M. L. A. \& Rédua, P. C. B. (2013). Manual de Referência para Procedimentos Clínicos em Odontopediatria (p. 25-36). Santos.

Nelson, T. M., Sheller, B., Friedman, C. S., \& Bernier, R. (2015). Educational and therapeutic behavioral approaches to providing dental care for patients with Autism Spectrum Disorder. Special care in dentistry: official publication of the American Association of Hospital Dentists, the Academy of Dentistry for the Handicapped, and the American Society for Geriatric Dentistry, 35(3), 105-113. https://doi.org/10.1111/scd.12101

Picciani, B. L. S., Santos, P. S. da S., Soares Junior, L. A. V., \& Santos, B. M. dos. (2019). Diretrizes para atendimento odontológico de pacientes sistemicamente comprometidos. São Paulo: Quintessence. https://repositorio.usp.br/item/002931591

Picciani, B. L., Dos Santos, B. M., Silva-Júnior, G. O., Marinho, M. A., Papa, E. G., Faria, M. D., Bastos, L. F., \& de Gouvêa, C. V. (2019). Contribution of benzodiazepines in dental care of patients with special needs. Journal of clinical and experimental dentistry, 11(12), 1170-1174. https://doi.org/10.4317/jced.56149

Rojas, V., Rivera, A. \& Nilo, N. (2019). Actualización en diagnóstico e intervención temprana del Trastorno del Espectro Autista [Update in diagnosis and early intervention of Autistic Spectrum Disorder]. Rev Chil Pediatr. 90(5), 478-484. http://dx.doi.org/10.32641/rchped.v90i5.1294

Stein, L. I., Polido, J. C., Mailloux, Z., Coleman, G. G., \& Cermak, S. A. (2011). Oral care and sensory sensitivities in children with autism spectrum disorders. Special care in dentistry: official publication of the American Association of Hospital Dentists, the Academy of Dentistry for the Handicapped, and the American Society for Geriatric Dentistry, 31(3), 102-110. https://doi.org/10.1111/j.1754-4505.2011.00187.x

Stein Duker, L. I., Floríndez, L. I., Como, D. H., Tran, C. F., Henwood, B. F., Polido, J. C., \& Cermak, S. A. (2019). Strategies for Success: A Qualitative Study of Caregiver and Dentist Approaches to Improving Oral Care for Children with Autism. Pediatric dentistry, 41(1), 4E-12E. https://pubmed.ncbi.nlm.nih.gov/30803480/

Volkmar, F. R.; \& Wiesner, L.A. (2019). Autismo: Guia essencial para compreensão e tratamento. Artmed. p. 1-24.

Zink, A. G., Diniz, M. B., Rodrigues Dos Santos, M. T., \& Guaré, R. O. (2016). Use of a Picture Exchange Communication System for preventive procedures in individuals with autism spectrum disorder: pilot study. Special care in dentistry: official publication of the American Association of Hospital Dentists, the Academy of Dentistry for the Handicapped, and the American Society for Geriatric Dentistry, 36(5), 254-259. https://doi.org/10.1111/scd.12183 